\title{
Fluorogenic ATP Analogues for Online Monitoring of ATP Consumption: Observing Ubiquitin Activation in Real Time**
}

\author{
Stephan M. Hacker, Dana Pagliarini, Thomas Tischer, Norman Hardt, Daniel Schneider, \\ Martin Mex, Thomas U. Mayer, Martin Scheffner,* and Andreas Marx*
}

The conjugation of ubiquitin to proteins plays an important role in the regulation of numerous cellular processes. ${ }^{[1]}$ Deregulation of this pathway has been associated with different human disorders including cancer and neurodege nerative diseases. ${ }^{[2]}$ For ubiquitylation, ubiquitin is initially activated by a ubiquitin activating enzyme (E1) at the expense of adenosine triphosphate (ATP; see Figure 1a) to form a thioester bond between the $\mathrm{C}$ terminal glycine of ubiquitin and the catalytic cysteine residue of E1. ${ }^{[3]}$ Subse quent transfer to a cysteine residue of a ubiquitin conjugating enzyme (E2) initiates the conjugation of ubiquitin to a target protein (mostly to a lysine residue by means of an isopeptide

a)
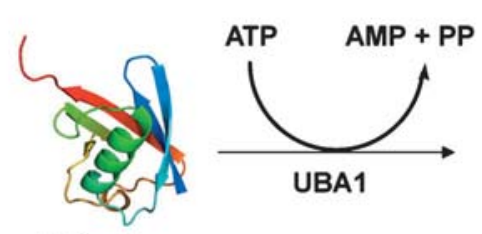

Ubiquitin

b)

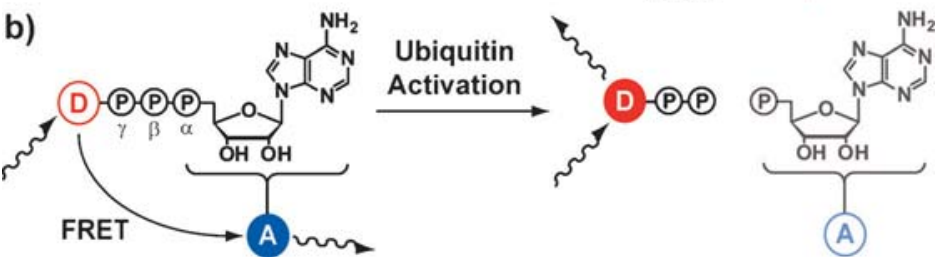

Figure 1. a) Mechanism of the activation of ubiquitin by UBA1. Ubiquitin is loaded onto UBA1 through the formation of a thioester bond with consumption of ATP. AMP: adenosine monophosphate, PP: pyrophosphate. b) Concept of signaling ATP consumption. The intact ATP analogue shows fluorescence of the acceptor (A) upon excitation of the donor (D) due to FRET. Upon cleavage by UBA1 direct donor fluorescence can be observed.

[*3] M. Sc. S. M. Hacker, Dipl. Biol. D. Pagliarini, Dipl. Biochem. T. Tischer, Dipl. Chem. N. Hardt, Dr. D. Schneider, B. Sc. M. Mex, Prof. Dr. T. U. Mayer, Prof. Dr. M. Scheffner, Prof. Dr. A. Marx

Departments of Chemistry and Biology, Konstanz Research School Chemical Biology, University of Konstanz

Universitätsstrasse 10, 78457 Konstanz (Germany)

E mail: martin.scheffner@uni konstanz.de andreas.marx@uni konstanz.de

[*:*] This work was supported financially by the Deutsche Forschungs gemeinschaft (SFB 969), the Studienstiftung des deutschen Volkes (stipend to S.M.H.), and the Konstanz Research School Chemical Biology. S.M.H. acknowledges support by the Zukunftskolleg of the University of Konstanz. bond), a process which in many cases requires the help of a ubiquitin ligase (E3). ${ }^{[3]}$ As UBA1 is one of only two known human E1 enzymes for ubiquitin, ${ }^{[4]}$ modulation of its activity may prove beneficial in the treatment of certain disorders. Hence, assays for studying the activation of ubiquitin by UBA1 directly and without the interfering effects of the downstream enzymatic cascade are important tools to analyze UBA1 activity and identify modulators of this enzyme.

So far, only a few assays that directly measure E1 activity have been described. ${ }^{[5]}$ However, these are laborious and not suitable for the continuous monitoring of E1 activity. In fact, until now no assay is available that allows the direct detection of ubiquitin activation in real time and that, for example, can be easily used to screen for E1 effectors. We, therefore, decided to elaborate on a conceptually novel assay: real time detection of ubiquitin activation by monitoring the cleavage of an ATP analogue (Figure 1b) that harbors two different fluorophores with the potential to undergo Förster resonance energy transfer (FRET). In the envisioned doubly labeled ATP analogue, excitation of the fluorescence donor (D) leads to transfer of the excitation energy to the fluorescence acceptor (A) whose fluorescence is monitored. Upon cleavage of the $\alpha / \beta$ anhydride bond of ATP, FRET is no longer possible and, thus, direct emission of the fluorescence donor can be detected. In this way, E1 activity results in a large change of fluorescence characteristics of the ATP analogue. Similar approaches based on the cleavage of FRET cassettes have been used for studying other hydro lyzing enzymes like proteases. ${ }^{[6]}$

For the envisaged time resolved ATPase sensor (TRASE) approach two fluorophores have to be attached to ATP. As the N6 position of ATP has been proven to be an attractive site for modifications without compromising UBA1 activity, ${ }^{[7]}$ the second modification has to be placed at the phosphoanhydride chain so that the two fluorophores are spatially separated upon cleavage. As earlier studies revealed that phosphate esters of ATP are stable, ${ }^{[8]}$ we tested $\gamma$ modified triphosphate II and $\delta$ modified tetraphosphate III in promoting the autoubiquity lation of E6AP, a reaction that involves UBA1 and the E2 enzyme UbcH5b (Figure 2a,b). E6AP is an E3 ligase that has been causally associated with the development of three different human disorders: cervical cancer, Angelman syn drome, and autism spectrum disorders. ${ }^{[9]}$ As only the UBA1 reaction is ATP dependent, the appearance of polyubiquity lated E6AP (E6AP Ub), which does not significantly migrate in the applied gel electrophoresis, ${ }^{[10]}$ and consequently 
a)
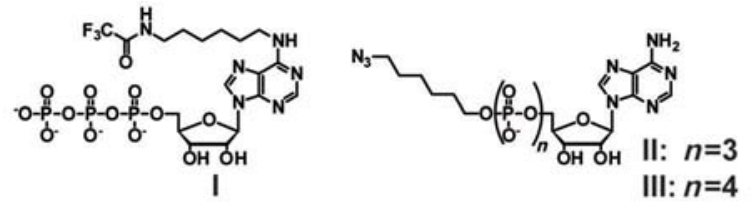

b)

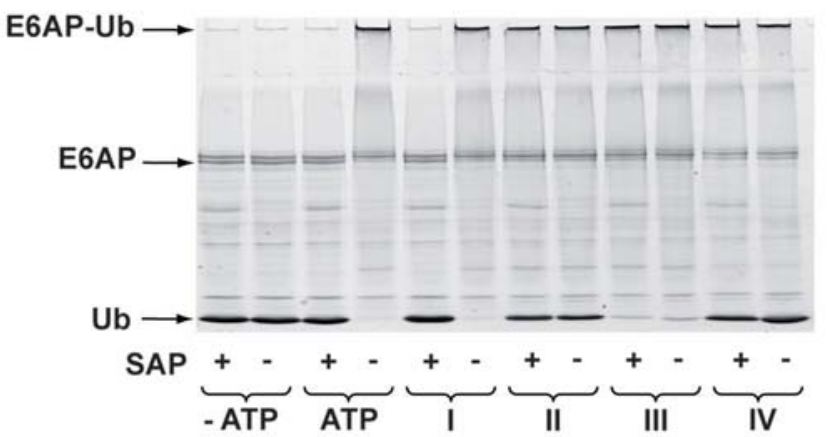

c)
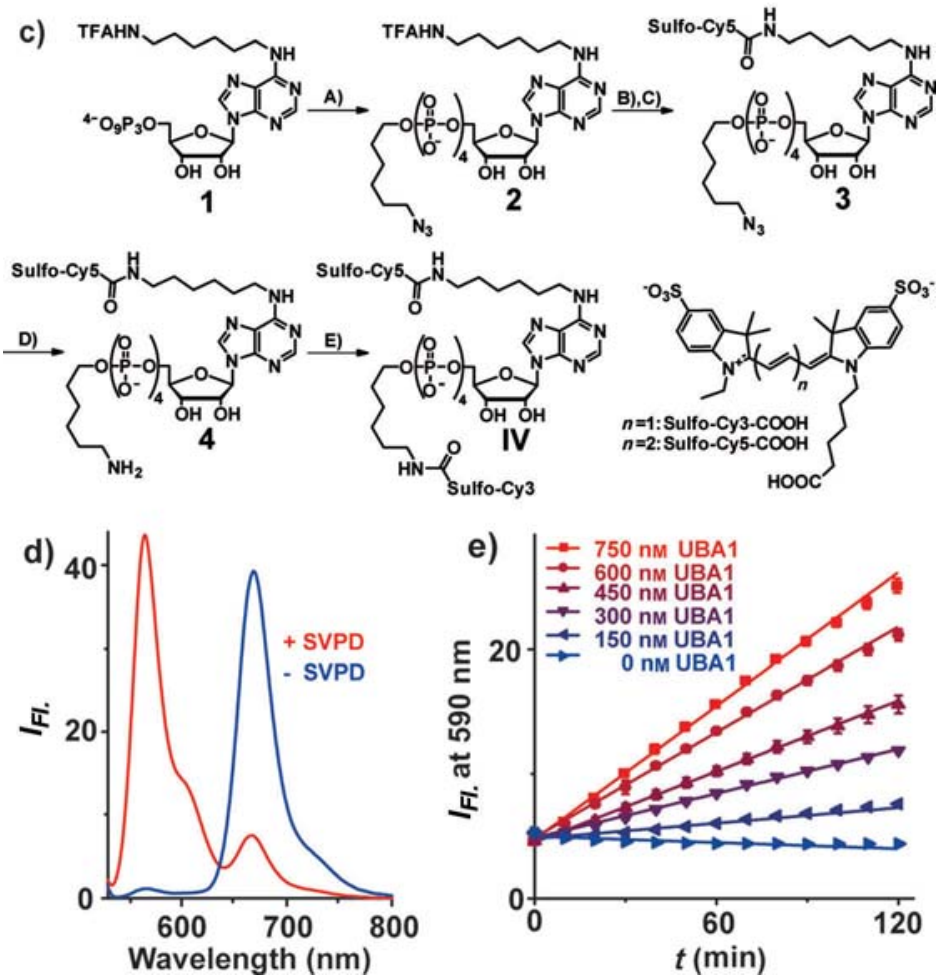

Figure 2. Ubiquitin activation by UBA1 is directly visualized in real time. a) Structures of ATP analogues used to explore the substrate scope of UBA1. b) Investigation of substrate scope of UBA1 for ATP analogues by the E6AP autoubiquitylation assay. UBA1 activity is monitored by the appearance of polyubiquitylated E6AP (E6AP Ub) and a decrease of the band intensity of ubiquitin (Ub) in a Coomassie stained SDS PAGE. The samples pretreated with shrimp alkaline phosphatase (SAP) are indicated. The experiment was repeated three times. c) Synthesis of the doubly labeled probe IV. A) 1. EDC hydrochlo ride, DMF, $2.5 \mathrm{~h}, \mathrm{RT} ; 2$. MeOH, $3 \mathrm{~h}, \mathrm{RT} ; 3.6$ azidohexylphosphate, $12 \mathrm{~h}, 40^{\circ} \mathrm{C}$, $23 \%$; B) $0.1 \mathrm{M} \mathrm{NaOH}, 4 \mathrm{~h}, \mathrm{RT}$; C) Sulfo Cy5 NHS ester, $0.1 \mathrm{M} \mathrm{NaHCO}_{3}, \mathrm{DMF}$, $\mathrm{pH} 8.7,12 \mathrm{~h}, \mathrm{RT}, 23 \%$ (over two steps); D) TCEP, Et $\mathrm{N}_{3}, \mathrm{H}_{2} \mathrm{O}, \mathrm{MeOH}, 4 \mathrm{~h}, \mathrm{RT}$, $74 \%$; E) Sulfo Cy3 NHS ester, $0.1 \mathrm{M} \mathrm{NaHCO}_{3}, \mathrm{DMF}, \mathrm{pH} 8.7,12 \mathrm{~h}, \mathrm{RT}, 61 \%$. d) Fluorescence spectra of the doubly labeled ATP analogue IV after incubation without (blue) and with (red) snake venom phosphodiesterase (SVPD), which quantitatively cleaves IV. e) Activation of ubiquitin by different concentrations of UBAI is monitored directly by the cleavage of probe IV using the fluorescence intensity of the fluorescence donor (D) as readout. Values represent mean \pm standard error of the mean of triplicates. decreased levels of free ubiquitin directly correlate with ATP consumption by UBA1. Interestingly it turned out that $\delta$ modified tetraphosphate III is a superior substrate for UBA1 than the corresponding triphosphate II as evidenced by the more efficient consumption of ubiquitin (Figure $2 b$ ). To validate these results, potential contaminations by unmodified ATP were removed by prior treatment with shrimp alkaline phosphatase (SAP), which does not hydrolyze II and III (Figure 2b). Again it was observed that III is more efficiently processed than II.

With this information in hand, we next synthesized the doubly labeled ATP analogue IV (Figure 2c). The fluorophores Sulfo Cy3 and Sulfo Cy5 were chosen, as they are especially well suited for FRET applications at short donor acceptor distances. ${ }^{[11]}$ Our synthesis started with triphosphate $\mathbf{1}^{[7]}$ Modification of the phosphate chain was accomplished by activating the triphosphate with 1 ethyl 3 (3 dimethylaminopropyl) carbodiimide (EDC) and subsequent addition of 6 azidohexylphosphate giving compound $\mathbf{2}$ in $23 \%$ yield. Cleavage of the trifluoroacetamide (TFA) protecting group with sodium hydroxide, followed by coupling of the Sulfo Cy5 $N$ hydroxysuccinimide (NHS) ester at $\mathrm{pH} 8.7$ resulted in the singly labeled molecule $\mathbf{3}$ in $23 \%$ yield. This could be further transformed by reduction of the azide with tris (2 carboxyethyl)phos phine (TCEP) giving compound $\mathbf{4}$ in $74 \%$ yield followed by coupling of the Sulfo Cy3 NHS ester to obtain final compound IV in a yield of $61 \%$.

After validation of the fluorescence properties of IV in a phosphodiesterase assay (Figure $2 \mathrm{~d}$ and Fig ure S1 in the Supporting Information), IV was tested as an ATP surrogate to be accepted by UBA1. Indeed, UBA1 used IV as a substrate as indicated by the appearance of ubiquitylated E6AP (Figure 2b). Anal yses of the levels of free ubiquitin revealed that far less ubiquitin was conjugated to E6AP in the presence of IV than in the presence of ATP. Furthermore, a titra tion experiment indicated that at least $250 \mu \mathrm{M} \mathrm{IV}$ is needed to reach a maximal reaction rate (Figure S2 in the Supporting Information). This shows that the $K_{\mathrm{M}}$ value of IV is also considerably higher than that of ATP $(4.7 \pm 1.0 \mu \mathrm{M}) \cdot{ }^{[12]}$ However, in view of the modi fication this is not unexpected.

Finally, we used IV to monitor ubiquitin activation by UBA1 in real time in the absence of any other enzyme. We incubated IV and ubiquitin with different concentrations of UBA1 in a 384 well format and followed the fluorescence signal (Figure 2e). We observed a time dependent linear increase of the fluorescence intensity of the fluorescence donor Sulfo Cy3 at $590 \mathrm{~nm}$ at all UBA1 concentrations tested. The rise in fluorescence correlated well with the concentration of UBA1 used (Figure S3 in the Supporting Information) demonstrating the practic ability of our approach.

Next, we checked if the ATP analogue IV opti mized for UBA1 can also be used to study other E1 
enzymes of ubiquitin like modifiers (Ubls). ${ }^{[13]}$ Therefore, we investigated the activation of NEDD8 by APPBP1/ $\mathrm{UBA} 3^{[13,14]}$ and SUMO1 by SAE1/SAE2 ${ }^{[13,15]}$ in the presence of IV (Figure 3a). Indeed, all three E1 enzymes can be studied by TRASE as indicated by the rise of fluorescence intensity in the presence of the cognate Ubls. Furthermore,

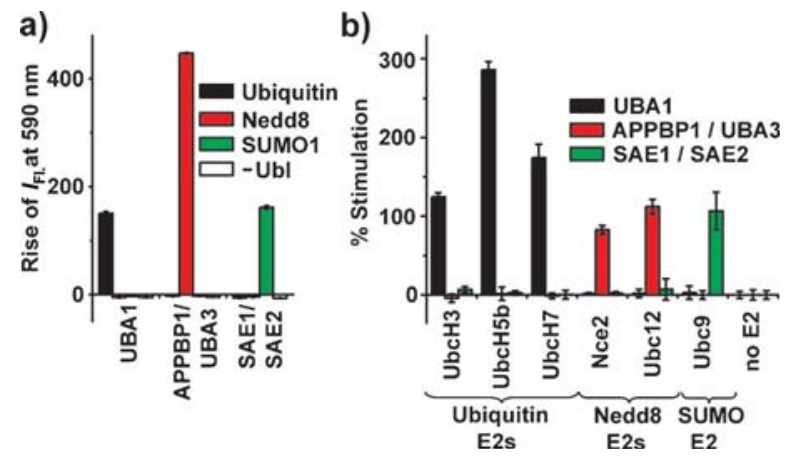

Figure 3. Scope of the method. a) Activation of ubiquitin and the Ubls SUMO1 and Nedd8 by cognate E1s. b) Stimulating effect of E2 enzymes on cognate Els. Rise of fluorescence was calculated by linear fitting of the fluorescence intensity over time. \% Stimulation was calculated from the rise of fluorescence at $590 \mathrm{~nm}$ in the presence of the respective E2 relative to the mean of the rise of the controls without E2. Values represent mean \pm standard error of the mean of triplicates.

E1 activity could only be observed when the correct E1 Ubl pair was used.

To further explore the applicability, we tested the potential influence of E2 enzymes on E1 activity. As shown in Figure $3 b$, we observed an increase in the activity of the different E1s in the presence of their cognate E2s, while the E1s were not stimulated by noncognate E2s. Thus, TRASE provides a simple tool to determine the specificities of E2s for E1 enzymes and in general, to identify positive and negative effectors of E1s.

Inhibitors of UBA1 have been proposed to be potentially useful in the treatment of certain types of cancer. ${ }^{[16]}$ However, only a few UBA1 inhibitors have been described, $\left.{ }^{[16} 18\right]$ which may be due to the fact that robust assays for directly monitoring UBA1 activity in a high throughput format have not been available. Indeed, previous screens to identify UBA1 inhibitors either relied on cell based assays that monitor the degradation of ubiquitylated target proteins ${ }^{[18]}$ or used the detection of ubiquitin chains in vitro. ${ }^{[19]}$ In these assays inhibition of all downstream enzymes of the ubiquitylation cascade has to be excluded during cumbersome hit validation. In contrast, TRASE monitors UBA1 activity directly (in the absence of any downstream enzyme or reagent) and, thus, appears to be ideally suited to identify specific UBA1 inhibitors. Furthermore, since the assay provides multiple time resolved data points, compounds that unspecifically affect the fluorescence characteristics of IV can be easily excluded.

To show the applicability of TRASE for identifying UBA1 inhibitors, we screened 1279 compounds that are either natural products or FDA approved drugs. This resulted in the identification of five hits that inhibit UBA1 activity to at least $90 \%$ (see also Figures S4 and S5 in the Supporting Information). Further characterization using the TRASE assay revealed that one compound, $\beta$ lapachone (Figure $4 \mathrm{a}$ ), inhibits $\mathrm{E} 1$ activity with an $\mathrm{IC}_{50}$ of $(1.62 \pm 0.13) \mu \mathrm{M}$ (Fig ure $4 \mathrm{~b}$ ). This compound is also able to inhibit autoubiqityla tion of E6AP with a similar $\mathrm{IC}_{50}$ of $(1.59 \pm 0.07) \mu \mathrm{M}$ (Figure $4 \mathrm{~b}$

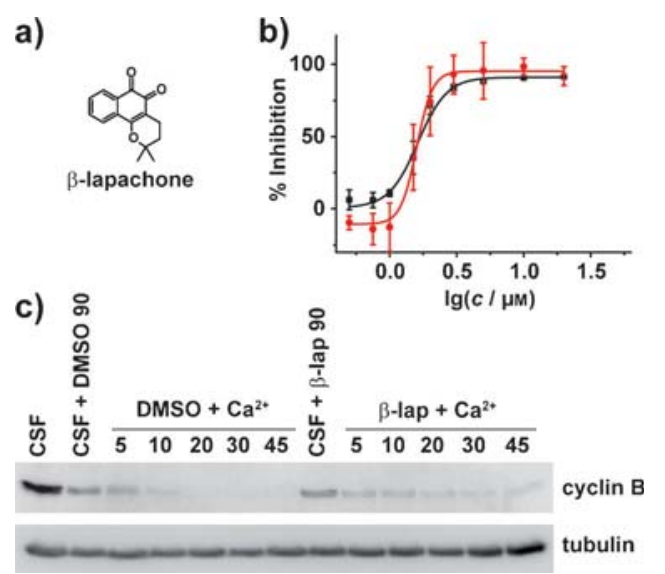

Figure 4. Inhibition of ubiquitin activation by $\beta$ lapachone. a) Structure of $\beta$ lapachone. b) Concentration dependence of UBA1 inhibition by $\beta$ lapachone measured by the TRASE (black squares) and the E6AP autoubiquitylation assay (red circles). Rise of fluorescence in the TRASE assay was calculated by linear fitting of the fluorescence intensity over time. Consumption of ubiquitin in the E6AP autoubiqui tylation assay was calculated by quantifying the ubiquitin band. $\%$ Inhibition was calculated from these values relative to the positive and negative controls. Values represent mean \pm standard error of the mean of triplicates. c) Calcium induced degradation of cyclin B in Xenopus laevis egg extract pretreated with DMSO or $500 \mu \mathrm{M} \beta$ lapachone. Time is given in minutes; samples were analyzed by immunoblotting for cyclin B. Ubiquitin dependent degradation of cyclin B is slowed down by $\beta$ lapachone, CSF: cell extract arrested in metaphase by cytostatin factor. Tubulin serves as loading control. The experiment was repeated three times. $\beta$ lap: $\beta$ lapachone.

and Figure S6 in the Supporting Information). $\beta$ Lapachone is known to have anticancerogenic and pro apoptotic effects on different cancer cell lines, ${ }^{[20]}$ but has not yet been associated with inhibition of the ubiquitylation pathway. To obtain evidence that $\beta$ lapachone interferes with $\mathrm{E} 1$ activity in a physiologically relevant setting, we decided to investigate the inhibitory potential of $\beta$ lapachone in Xenopus laevis egg extract, ${ }^{[21]}$ which represents a valuable system to study the function of the ubiquitin pathway in regulating cell cycle progression. The metaphase arrested extract was pretreated with $\beta$ lapachone or DMSO in an experimental setup that is commonly used for these kinds of assays. ${ }^{[22]}$ By addition of calcium, the metaphase arrest of the extract was relieved resulting in the activation of the E3 ubiquitin ligase Anaphase Promoting Complex/Cyclosome (APC/C) ${ }^{[23]}$ and, conse quently, in the destruction of cyclin B. Indeed, in the presence of DMSO, cyclin B levels decreased indicating that it was ubiquitylated and subjected to proteasomal degradation (Figure $4 \mathrm{c}$ ). In contrast, the signal for cyclin B remained detectable for a prolonged time in the extract treated with $\beta$ 
lapachone. Thus, these data suggest that $\beta$ lapachone prevents the destruction of cyclin B by inhibiting the loading of E1 with ubiquitin in Xenopus laevis egg extracts.

In summary, with TRASE we present a conceptually new method to monitor the activity of the ATP consuming enzyme UBA1. The method is based on fluorogenic ATP analogues undergoing a large change in fluorescence charac teristics upon enzymatic processing. The method can be used to monitor and quantify the activation of ubiquitin and ubiquitin like proteins by their cognate E1s in the absence of the downstream enzyme cascade in real time in a facile and parallel fashion. Additionally, the presented approach is applicable to study effects of protein interactions on activity as exemplarily shown for E1 E2 interactions. This opens the perspective, for example, of using TRASE to screen for small molecules acting on E2 enzymes. Furthermore, we show that the method is well suited for high throughput screening endeavors and identified $\beta$ lapachone as a novel inhibitor of UBA1. Finally, the presented assay may not only be useful for identifying positive and negative effectors of E1 enzymes, but also holds the potential for studying other ATP cleaving enzymes.

Keywords: ATPases · FRET · lapachone $\cdot$ nucleotides $\cdot$ ubiquitin

[1] a) T. Ravid, M. Hochstrasser, Nat. Rev. Mol. Cell Biol. 2008, 9 679; b) R. L. Welchmann, C. Gordon, R. J. Mayer, Nat. Rev. Mol. Cell Biol. 2005, 6, 599 .

[2] a) D. Rotin, S. Kumar, Nat. Rev. Mol. Cell Biol. 2009, 10, 398 b) R. J. Deshaies, C. A. P. Joazeiro, Annu. Rev. Biochem. 2009, $78,399$.

[3] a) A. Hershko, A. Ciechanover, Annu. Rev. Biochem. 1998, 67, 425; b) D. T. Huang, H. Walden, D. Duda, B. A. Schulman, Oncogene 2004, 23, 1958; c) B. A. Schulman, J. W. Harper, Nat. Rev. Mol. Cell Biol. 2009, 10, 319.

[4] a) J. Jin, X. Li, S. P. Gygi, J. W. Harper, Nature 2007, 447, 1135; b) C. Pelzer, I. Kassner, K. Matentzoglu, R. K. Singh, H. P. Wollscheid, M. Scheffner, G. Schmidtke, M. Groettrup, J. Biol. Chem. 2007, 282, 23010.

[5] a) A. Hershko, H. Heller, S. Elias, A. Ciechanover, J. Biol. Chem. 1983, 258, 8206; b) A. Ciechanover, H. Heller, R. Katz Etzion, A. Hershko, Proc. Natl. Acad. Sci. USA 1981, 78, 761; c) C. E. Berndsen, C. Wolberger, Anal. Biochem. 2011, 418, 102.

[6] a) A. C. Correa, C. Schulz in Laboratory Techniques in Bio chemistry and Molecular Biology: FRET and FLIM techniques, Vol. 15 (Ed.: T. W. J. Gadella), Elsevier, Oxford, 2009, p. 225; b) C. Lombard, J. Saulnier, J. Wallach, Biochimie 2005, 87, 265; c) V. V. Didenko, Biotechniques 2001, 31, 1106; d) E. D. Mata yoshi, G. T. Wang, G. A. Krafft, J. Erickson, Science 1990, 247, 954; e) R. T. Cummings, S. P. Salowe, B. R. Cunningham, J. Wiltsie, Y. W. Park, L. M. Sonatore, D. Wisniewski, C. M. Douglas, J. D. Hermes, E. M. Scolnick, Proc. Natl. Acad. Sci. USA 2002, 99, 6603; f) O. Wichmann, J. Wittbrodt, C. Schulz, Angew. Chem. 2006, 118, 522; Angew. Chem. Int. Ed. 2006, 45, 508 .

[7] S. M. Hacker, N. Hardt, A. Buntru, D. Pagliarini, M. Möckel, T. U. Mayer, M. Scheffner, C. R. Hauck, A. Marx, Chem. Sci. 2013, 4, 1588.

[8] S. M. Hacker, M. Mex, A. Marx, J. Org. Chem. 2012, 77, 10450.

[9] M. Scheffner, S. Kumar, Biochim. Biophys. Acta 2013, DOI: 10.1016/j.bbamcr.2013.03.024 (available online PMID: 23545411).

[10] U. Nuber, S. E. Schwarz, M. Scheffner, Eur. J. Biochem. 1998, $254,643$.

[11] A. Dietrich, V. Buschmann, C. Müller, M. Sauer, Rev. Mol. Biotechnol. 2002, 82, 211.

[12] K. E. Wee, Z. Lai, K. R. Auger, J. Ma, K. Y. Horiuchi, R. L. Dowling, C. S. Dougherty, J. I. Corman, R. Wynn, R. A. Cope land, J. Protein Chem. 2000, 19, 489.

[13] A. G. van der Veen, H. L. Ploegh, Annu. Rev. Biochem. 2012, 81, 323.

[14] F. Osaka, H. Kawasaki, N. Aida, M. Saeki, T. Chiba, S. Kawashima, K. Tanaka, S. Kato, Genes Dev. 1998, 12, 2263.

[15] T. Okuma, R. Honda, G. Ichikawa, N. Tsumagari, H. Yasuda, Biochem. Biophys. Res. Commun. 1999, 254, 693.

[16] S. R. da Silva, S. L. Paiva, J. L. Lukkarila, P. T. Gunning, J. Med. Chem. 2013, 56, 2165 .

[17] a) J. J. Chen et al., J. Biol. Chem. 2011, 286, 40867; b) Y. Yang et al., Cancer Res. 2007, 67, 9472; c) S. Tsukamoto, H. Hirota, M. Imachi, M. Fujimuro, H. Onuki, T. Ohtaa, H. Yokosawae, Bioorg. Med. Chem. Lett. 2005, 15, 191; d) D. Ungermannova, S. J. Parker, C. G. Nasveschuk, W. Wang, B. Quade, G. Zhang, R. D. Kuchta, A. J. Phillips, X. Liu, PLoS One 2012, 7, e29208; e) M. Matsuzawa, H. Kakeya, J. Yamaguchi, M. Shoji, R. Onose, H. Osada, Y. Hayashi, Chem. Asian J. 2006, 1, 845.

[18] D. Ungermannova, S. J. Parker, C. G. Nasveschuk, D. A. Chap nick, A. J. Phillips, R. D. Kuchta, X. Liu, J. Biomol. Screening 2012, 17, 421.

[19] I. V. Davydov, D. Woods, Y. J. Safiran, P. Oberoi, H. O. Fearn head, S. Fang, J. P. Jensen, A. M. Weissman, J. H. Kenten, K. H. Vousden, J. Biomol. Screening 2004, 9, 695.

[20] Y. Li, X. Sun, J. T. LaMont, A. B. Pardee, C. J. Li, Proc. Natl. Acad. Sci. USA 2003, 100, 2674.

[21] A. W. Murray, Methods Cell. Biol. 1991, 36, 581.

[22] a) T. Tischer, E. Hörmanseder, T. U. Mayer, Science 2012, 338, 520; b) E. Hörmanseder, T. Tischer, S. Heubes, O. Stemmann, T. U. Mayer, EMBO Rep. 2011, 12, 436; c) M. Gaffre, A. Martoriati, N. Belhachemi, J. P. Chembon, E. Houliston, C. Jessus, A. Karaiskou, Development 2011, 138, 3735.

[23] N. R. Rauh, A. Schmidt, J. Bormann, E. A. Nigg, T. U. Mayer, Nature 2005, 437, 1048. 